\title{
Effects of acute autonomic modulation on atrial conduction delay and local electrograms duration in paroxysmal atrial fibrillation
}

\author{
Mário Oliveira a,*, Nogueira da Silva a , Pedro Cunha ${ }^{a}$, Ruben Ramos ${ }^{a}$, Fernando Marques a , Sofia Santos ${ }^{\text {a }}$, \\ Isabel Rocha ${ }^{\mathrm{b}}$, Luis Silva-Carvalho ${ }^{\mathrm{b}}$, Rui Ferreira ${ }^{\mathrm{a}}$ \\ a Cardiology Department, Santa Marta Hospital, Lisbon, Portugal \\ b Institute of Physiology, Faculty of Medicine, Lisbon, Portugal
}

\section{A R T I C L E I N F O}

Article history:

Received 30 July 2009

Received in revised form 2 December 2009

Accepted 4 February 2010

Available online 17 March 2010

\section{Keywords:}

Paroxysmal atrial fibrillation

Atrial conduction

Electrograms

Autonomic nervous system

\begin{abstract}
A B S T R A C T
Slowed atrial conduction may contribute to reentry circuits and vulnerability for atrial fibrillation (AF). The autonomic nervous system (ANS) has modulating effects on electrophysiological properties. However, complex interactions of the ANS with the arrhythmogenic substrate make it difficult to understand the mechanisms underlying induction and maintenance of AF.

Aim: To determine the effect of acute ANS modulation in atrial activation times in patients (P) with paroxysmal AF (PAF).

Methods and results: $16 \mathrm{P}$ (9 men; $59 \pm 14$ years) with PAF, who underwent electrophysiological study before $\mathrm{AF}$ ablation, and 15P ( 7 men; $58 \pm 11$ years) with atrioventricular nodal reentry tachycardia, without documentation or induction of AF (control group). Each group included 7P with arterial hypertension but without underlying structural heart disease. The study was performed while off drugs. Multipolar catheters were placed at the high right atrium (HRA), right atrial appendage (RAA), coronary sinus (CS) and His bundle area (His). At baseline and with HRA pacing (600 ms, shortest propagated S2) we measured: i) intraatrial conduction time (IACT, between RAA and atrial deflection in the distal His), ii) inter-atrial conduction time (interACT, between RAA and distal CS), iii) left atrial activation time (LAAT, between atrial deflection in the distal His and distal CS), iv) bipolar electrogram duration at four atrial sites (RAA, His, proximal and distal CS). In the PAF group, measurements were also determined during handgrip and carotid sinus massage (CSM), and after pharmacological blockade of the ANS (ANSB). AF was induced by HRA programmed stimulation in $56 \%$ (self-limited -6 ; sustained -3 ), $68.8 \%$ (self-limited -6 ; sustained -5 ), and $50 \%$ (selflimited -5 ; sustained -3 ) of the $\mathrm{P}$, in basal, during ANS maneuvers, and after ANSB, respectively $(p=N S)$. IACT, interACT and LAAT significantly lengthened during HRA pacing in both groups (600 ms, S2). P with PAF have longer IACT $(p<0.05)$, a higher increase in both IACT, interACT $(p<0.01)$ and electrograms duration $(p<0.05)$ with S2, and more fragmented activity, compared with the control group. Atrial conduction times and electrograms duration were not significantly changed during ANS stimulation. Nevertheless, ANS maneuvers increased heterogeneity of the local electrograms duration. Also, P with sustained AF showed longer interACT and LAAT during CSM.

Conclusion: Atrial conduction times, electrograms duration and fractionated activity are increased in PAF, suggesting a role for conduction delays in the arrhythmogenic substrate. Acute vagal stimulation is associated with prolonged interACT and LAAT in P with inducible sustained AF and ANS modulation may influence the heterogeneity of atrial electrograms duration.
\end{abstract}

(c) 2010 Elsevier Ireland Ltd. All rights reserved.

\section{Introduction}

Atrial fibrillation (AF) is the most common cardiac arrhythmia in clinical practice. It has been recognized as a growing problem, with a prevalence ranging from $1 \%$ in the general population to more than $5 \%$ over the age of $65[1,2]$. The complex pathophysiology of AF has not been

\footnotetext{
* Corresponding author. Cardiology Department, Santa Marta Hospital, Rua Santa Marta, 1169-024 Lisbon, Portugal. Tel.: + 351 213594311; fax: + 351213560368. E-mail address: m.martinsoliveira@gmail.com (M. Oliveira).
}

clearly elucidated, due to limitations in studying the mechanisms that lead to the initiation and maintenance of this arrhythmia. Clinical and experimental works have provided new insights into a better understanding of AF, suggesting an important contribution of multiple depolarization wavelets, single dominant reentry circuits, focal sources of electrical activity, and different forms of atrial remodeling to the creation of electrophysiologic substrate for both the recurrence and progression to sustained AF [3-5]. Patients with established AF have regions of slowed conduction facilitating the functional substrate for the occurrence of reentry circuits within the atria [6]. 
Furthermore, atrial remodeling causes changes in atrial refractoriness and atrial conduction that may promote AF [7]. Autonomic nervous system (ANS) activity is believed to play an important role in $\mathrm{AF}$ pathogenesis $[8,9]$. The onset of $\mathrm{AF}$ is often preceded by fluctuations in autonomic balance that are recognized as modulators in mediating AF [10,11]. Also, a number of electrophysiological properties related with vulnerability for AF may change as a result of vagal or sympathetic activation $[12,13]$. Vagal stimulation reduces velocity of the conduction in the atrial tissue and shortens the atrial effective refractory periods (ERP) heterogeneously, whereas sympathetic stimulation can increase atrial conduction velocity, favor trigger activity and uniformly reduce atrial refractoriness. However, complex interactions of the ANS with the arrhythmogenic substrate make it difficult to understand its influence in the mechanisms underlying induction and maintenance of AF. The present study was performed to assess the effects of acute ANS modulation in atrial conduction times and the duration of atrial local electrograms in patients with paroxysmal $\mathrm{AF}$ (PAF).

\section{Methods}

\subsection{Patient groups}

The study included a group of 16 patients ( 9 men and 7 women with a mean age of $59 \pm 14$ years) with $\geq 1$ year duration of clinical history of PAF, documented with electrocardiograms and/or Holter recordings, despite antiarrhythmic therapy, referred to our institution for AF ablation, and a control group of 15 patients $(7$ men and 8 women with a mean age of $58 \pm 11$ years), with clinically documented supraventricular tachycardia (all with electrophysiological diagnosis of atrioventricular nodal reentry tachycardia). None of these patients had a history of AF or induction of AF during the electrophysiological study (EPS) performed before ablation. Each group included 7 patients with arterial hypertension, but without underlying structural heart disease assessed with transthoracic echocardiography.

Patients with previous myocardial infarction or angina, heart failure, evidence of sick sinus syndrome, failure to remain in stable sinus rhythm while in-hospital monitoring before the EPS, permanent pacemaker implanted, bronchopulmonary disease, sleep apnea, and pregnancy or thyroid dysfunction were excluded. Prior to the EPS, all antiarrhythmic drugs were withdrawn for at least 5 half-life times. Patients under amiodarone stopped treatment 2 months before the EPS. The study protocol was approved by the local ethics and performed according to the ethical guidelines of the Declaration of Helsinki. All subjects were required to give written informed consent.

\subsection{Electrophysiological protocol}

All patients underwent EPS in a non-sedated post-absorptive state. No serum electrolyte disturbances were found. Atrial electrical stimulation and recording of electrograms were performed by using $6 \mathrm{~F}$ bipolar catheter electrodes inserted percutaneously into the femoral and internal jugular veins. Quadripolar electrode catheters (2-mm-spaced; DaigCo) were positioned in the high anterior wall of the right atrium (HRA), right atrial appendage (RAA), His bundle area (HBE), and a decapolar catheter with $2 \mathrm{~mm}$ interelectrode distance and $5 \mathrm{~mm}$ space between each electrode pair was advanced into the coronary sinus (CS) as distal as possible. Stability of the electrode catheters was maintained by fluoroscopic monitoring. Surface ECG leads I, II, V1, and V5 and four intracardiac electrograms (RAA, HBE, CS proximal and CS distal) were displayed on an oscilloscope and a multichannel electrophysiological recorder (Bard Lab System) with a frequency response of $50-500 \mathrm{~Hz}$ used onto optical disks for later analysis.

Intra-atrial conduction time (IACT), the interval from the RAA to the atrial electrogram at the HBE, interatrial conduction time (interACT), the interval from the RAA to the atrial electrogram at the distal part of the CS, left atrial activation time (LAAT), the interval from the atrial electrogram at the HBE to the atrial electrogram at distal CS, and local wave duration from different atrial sites (RAA, HBE, proximal and distal CS) were obtained during sinus rhythm, at baseline drive-train stimulation (S1-S1, cycle length of $600 \mathrm{~ms}$ ) and at the earliest propagated extra-stimulus (S2) during S1 pacing at the HRA. The maximal prolongation of the atrial electrograms during S2 was represented by the \% of increase compared to baseline at each recording site.

Stimulation was performed with impulses of $2 \mathrm{~ms}$ duration at twice the diastolic threshold. All atrial electrograms were recorded at a fixed gain setting (accompanied by a $0.2 \mathrm{mV}=0.3 \mathrm{~mm}$ calibration signal) and remained almost consistent and reproducible at each recording site in each patient. The duration of the local electrograms was measured from the beginning of the earliest electrical activity that deviated from the stable baseline value to the last point of the atrial electrogram at which the baseline value was crossed [14]. Fragmented activity was defined as a disorganised atrial electrogram, with multiple deflections, resulting in a prolonged duration of the activation complex greater than or equal to $150 \%$ of the electrogram duration of basic beats [15].
Table 1

Baseline patient clinical characteristics and left atrial size.

\begin{tabular}{lll}
\hline Characteristic & PAF group $(n=16)$ & Control group $(n=15)$ \\
\hline Age, years & $59 \pm 14$ & $58 \pm 11$ \\
Male gender & $56 \%$ & $47 \%$ \\
Body mass index & $27 \pm 5$ & $28 \pm 6$ \\
History of hypertension & $44 \%$ & $47 \%$ \\
Heart rate, bpm & $65 \pm 9$ & $63 \pm 8$ \\
Systolic blood pressure, $\mathrm{mm} \mathrm{Hg}$ & $132 \pm 20$ & $126 \pm 21$ \\
Diastolic blood pressure, mm Hg & $85 \pm 11$ & $81 \pm 12$ \\
LA M-mode, mm & $43 \pm 3$ & $40 \pm 3$ \\
History of palpitations; years & $2.5 \pm 2.0$ & $3.0 \pm 2.0$
\end{tabular}

$\mathrm{PAF}=$ paroxysmal atrial fibrillation; $\mathrm{LA}=$ left atrium (M-mode measurements in parasternal view).

None of the variables differed significantly between the groups.

In the PAF group, measurements were made also during ANS stimulation maneuvers and after pharmacological ANS blockade (atropine $0.04 \mathrm{mg} / \mathrm{kg}+$ propranolol $0.15 \mathrm{mg} / \mathrm{kg}$ ). Sympathetic stimulation was achieved by $3 \mathrm{~min}$ of static, intermittent handgrip (HG) of submaximal intensity until fatigue set in, and vagal activity was induced by right carotid sinus massage (CSM), with pressure applied at the point of strongest pulse at the level of the cricoid cartilage (for $10 \mathrm{~s}$ at 10-s intervals in 3-min periods). In the absence of a response, CSM was repeated on the left side. Continuous ECG and blood pressure monitoring, together with spectral analysis of RR intervals in the frequency domain (Task Force Monitor 3040; CNSystems), were used to confirm ANS stimulation or blockade. The frequency spectrum was divided into three components: very low frequency (VLF) $(0-0.04 \mathrm{~Hz})$, low frequency (LF) $(0.04-0.15 \mathrm{~Hz})$ and high frequency (HF) $(0.15-0.4 \mathrm{~Hz})$. HF values, attributed to vagal modulation, are affected by mechanical stimulation of the carotid sinus, while LF values mainly reflect sympathetic activity and increase during HG. Intravenous administration of propranolol and atropine resulted in total suppression of HF and LF activity, thus enabling assessment of the intrinsic electrophysiological properties [16].

All patients underwent programmed bipolar stimulation (drive-train cycle length of 600 ms using S2-S3 extra-stimuli delivered after eight paced beats) and incremental pacing protocols (short-term of burst pacing range from 600 to $300 \mathrm{~ms}$ ) during sinus rhythm, by pacing from the distal electrode pair positioned at the HRA. AF was defined as a rapid atrial rhythm (rate $>350$ beats/min) characterized by variability of the beat-to-beat cycle length, polarity, configuration and amplitude of the recorded atrial electrograms and lasting more than 5 cycles [17]. AF was considered not inducible; inducible, self-limited ( $<60 \mathrm{~s}$ ) or inducible, sustained, terminated by therapeutic intervention [18].

\subsection{Statistical analysis}

Categorical variables are expressed as frequencies and percentages. Continuous variables were expressed as means \pm standard. Student's $t$ test was used to compare all paired data in the same group. Comparisons between groups were made using the unpaired Student's $t$ test, repeated ANOVA for continuous variables (overall comparison) or Mann-Whitney's test as appropriate. The chi-square test with Yates correction was used for categorical variables. A value of $p<0.05$ was considered statistically significant. Data were analyzed using GraphPAD Instruments version 3.05 (GraphPad Software, Inc., California, USA).

\section{Results}

Table 1 shows the clinical characteristics and the left atrial size (evaluated by M-mode echocardiography) of the patients with and without PAF. There were no significant differences between the groups.

Table 2

Comparison of the atrial conduction times between groups.

\begin{tabular}{llll}
\hline & SR & $600 \mathrm{~ms}$ & S2 \\
\hline IACT (ms) & & & \\
$\quad$ PAF group & $\psi 34 \pm 15$ & $52 \pm 19^{*}$ & $\S 102 \pm 45^{* *}$ \\
$\quad$ Control group & $24 \pm 13$ & $50 \pm 22^{*}$ & $63 \pm 19^{*}$ \\
InterACT (ms) & & & \\
$\quad$ PAF group & $82 \pm 19$ & $118 \pm 22^{* *}$ & $\S 176 \pm 52^{* *}$ \\
Control group & $73 \pm 20$ & $115 \pm 20^{* *}$ & $126 \pm 22^{* *}$ \\
LAAT (ms) & & & \\
PAF group & $53 \pm 15$ & $68 \pm 17^{*}$ & $77 \pm 58^{*}$ \\
Control group & $49 \pm 18$ & $58 \pm 19^{*}$ & $70 \pm 18^{*}$ \\
\hline
\end{tabular}

$\mathrm{SR}=$ sinus rhythm; $600 \mathrm{~ms}=$ drive-train stimulation with a cycle length of $600 \mathrm{~ms}$; $\mathrm{S} 2$ = the earliest propagated extra-stimulus; IACT = intra-atrial conduction time; interACT $=$ interatrial conduction time; LAAT $=$ left atrial activation time; PAF $=$ paroxysmal atrial fibrillation; control $=$ no clinical history of atrial fibrillation nor induction of atrial fibrillation. ${ }^{*} p<0.05$ (vs. measurement in SR); ${ }^{* *} p<0.01$ (vs. measurement in SR); ${ }^{\psi} p<0.05$ (between groups); ${ }^{\S} p<0.01$ (between groups). 
AF was induced during programmed stimulation in $56 \%$ (self-limited -6 ; sustained - 3), 68.8\% (self-limited - 6; sustained - 5), and 50\% (self-limited - 5; sustained -3 ) of the PAF group in basal, during ANS maneuvers, and after ANS blockade, respectively ( $p=\mathrm{NS}$ ).

In baseline, mean P-wave duration was $108 \pm 14 \mathrm{~ms}$ in patients with PAF and $96 \pm$ $20 \mathrm{~ms}$ in patients without $\mathrm{AF}(p=0.05)$. Baseline IACT, during sinus rhythm, was longer in the PAF group, compared to the control group, without significant differences in interACT and LAAT between groups (Table 2). IACT, interACT and LAAT significantly lengthened in both groups during HRA pacing (drive-train with a cycle length of $600 \mathrm{~ms}$ and during premature stimulation). Patients with PAF showed a greater prolongation in both IACT and interACT with the earliest propagated extra-stimulus $(p<0.01)$ (Table 2).

Electrogram duration showed significant differences at the RAA, when comparing both groups during baseline sinus rhythm and with S2 (Fig. 1). Also, patients with
PAF had greater prolongation of electrogram wave duration measured at the RAA and distal CS during the earliest propagated S2 ( $73 \pm 35 \%$ vs. $11 \pm 8 \%$ at the RAA and $13 \pm$ $3 \%$ vs. $1 \pm 6 \% \mathrm{~ms}$ at the distal CS, for PAF patients and control group, respectively; $p<0.05)$. Fragmented atrial activity was identified in $43.8 \%$ of the PAF group and in $6.7 \%$ of the control group $(p=0.03)$.

\subsection{Conduction parameters during acute autonomic modulation}

Table 3 summarizes the results of the atrial conduction intervals during HG and CSM maneuvers, and after ANS blockade among patients with PAF. Atrial conduction times were not significantly changed during ANS stimulation. However, patients with inducible sustained AF had longer interACT and LAAT during CSM.

Despite longer electrograms in RAA and His during CSM when compared to baseline recordings, there were no significant differences in the mean duration of the

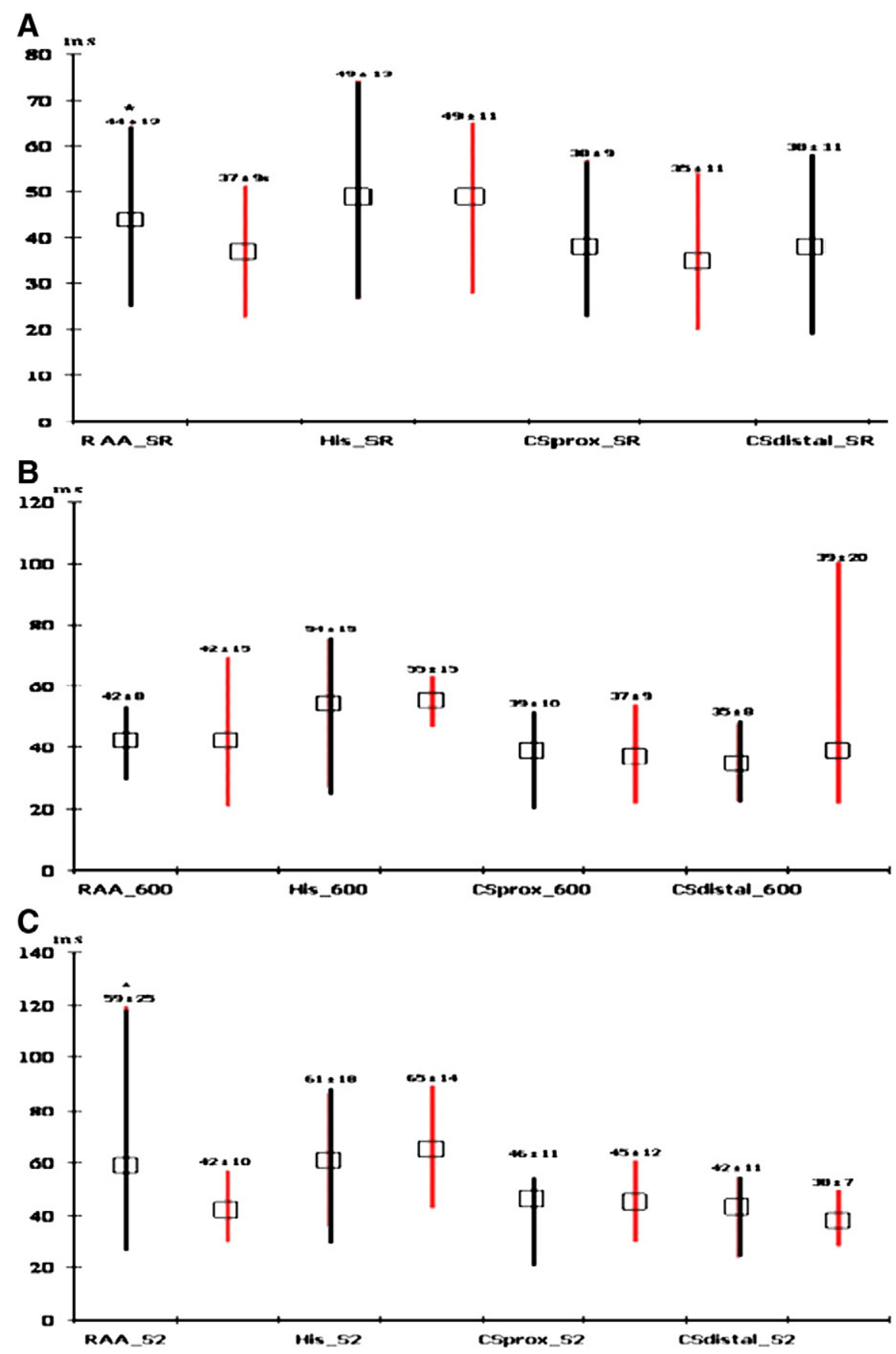

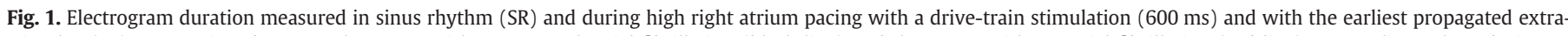

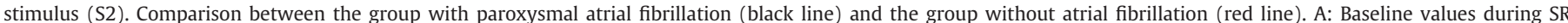

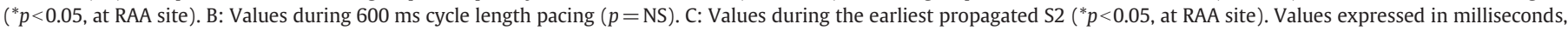

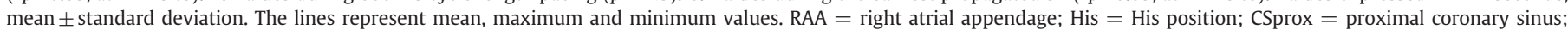
CSdistal $=$ distal coronary sinus . 
Table 3

Atrial conduction times during autonomic modulation in paroxysmal atrial fibrillation patients.

\begin{tabular}{|c|c|c|c|c|c|}
\hline & $\begin{array}{l}\text { PAF group } \\
(n=16)\end{array}$ & $\begin{array}{l}\text { AF non-inducible } \\
(n=5)\end{array}$ & $\begin{array}{l}\text { AF inducible } \\
(n=11)\end{array}$ & $\begin{array}{l}\text { AF self-limited } \\
(n=6)\end{array}$ & $\begin{array}{l}\text { AF sustained } \\
(n=5)\end{array}$ \\
\hline IACT_baseline & $34 \pm 15$ & $34 \pm 18$ & $35 \pm 15$ & $35 \pm 19$ & $35 \pm 10$ \\
\hline IACT_hand-grip & $29 \pm 11$ & $32 \pm 15$ & $28 \pm 10$ & $27 \pm 13$ & $29 \pm 7$ \\
\hline IACT_carotid sinus massage & $30 \pm 14$ & $29 \pm 7$ & $29 \pm 11$ & $30 \pm 9$ & $30 \pm 13$ \\
\hline IACT_ANS blockade & $28 \pm 13$ & $25 \pm 13$ & $30 \pm 14$ & $29 \pm 5$ & $30 \pm 13$ \\
\hline interACT_baseline & $82 \pm 19$ & $77 \pm 24$ & $83 \pm 19$ & $80 \pm 20$ & $86 \pm 20$ \\
\hline interACT_hand-grip & $84 \pm 20$ & $83 \pm 18$ & $84 \pm 22$ & $80 \pm 26$ & $89 \pm 17$ \\
\hline interACT_carotid sinus massage & $80 \pm 21$ & $76 \pm 28$ & $81 \pm 18$ & $71 \pm 17^{*}$ & $95 \pm 5^{*}$ \\
\hline interACT_ANS blockade & $72 \pm 14$ & $64 \pm 16$ & $80 \pm 8$ & $74 \pm 20$ & $77 \pm 9$ \\
\hline LAAT_baseline & $53 \pm 15$ & $44 \pm 7$ & $54 \pm 19$ & $53 \pm 10$ & $57 \pm 19$ \\
\hline LAAT_hand-grip & $55 \pm 17$ & $51 \pm 11$ & $57 \pm 19$ & $52 \pm 21$ & $62 \pm 16$ \\
\hline LAAT_carotid sinus massage & $52 \pm 16$ & $45 \pm 7^{* *}$ & $55 \pm 14$ & $47 \pm 7^{* *}$ & $65 \pm 14^{* *}$ \\
\hline LAAT_ANS blockade & $44 \pm 12$ & $38 \pm 11$ & $42 \pm 14$ & $45 \pm 13$ & $48 \pm 11$ \\
\hline
\end{tabular}

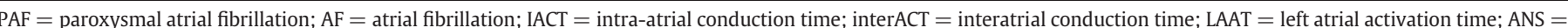
autonomic nervous system. Values expressed in milliseconds, mean \pm standard deviation. ${ }^{*} p<0.05 ;{ }^{* *} p<0.01$.

measured electrograms during autonomic stimulation or after autonomic blockade (Fig. 2). Nevertheless, we observed an increased heterogeneity of the atrial wave duration, with significant differences between the recording sites, appearing during ANS maneuvers, and abolished after ANS blockade (Fig. 2). Representative intracardiac electrograms, obtained from the RAA during sinus rhythm, with S2, HG and CSM, and after ANSB are shown in Fig. 3.

\section{Discussion}

Although the triggers for AF initiation appear to be located in the pulmonary veins, established $\mathrm{AF}$ has been associated with conduction disturbances and heterogeneous reduction of ERP, that facilitate the

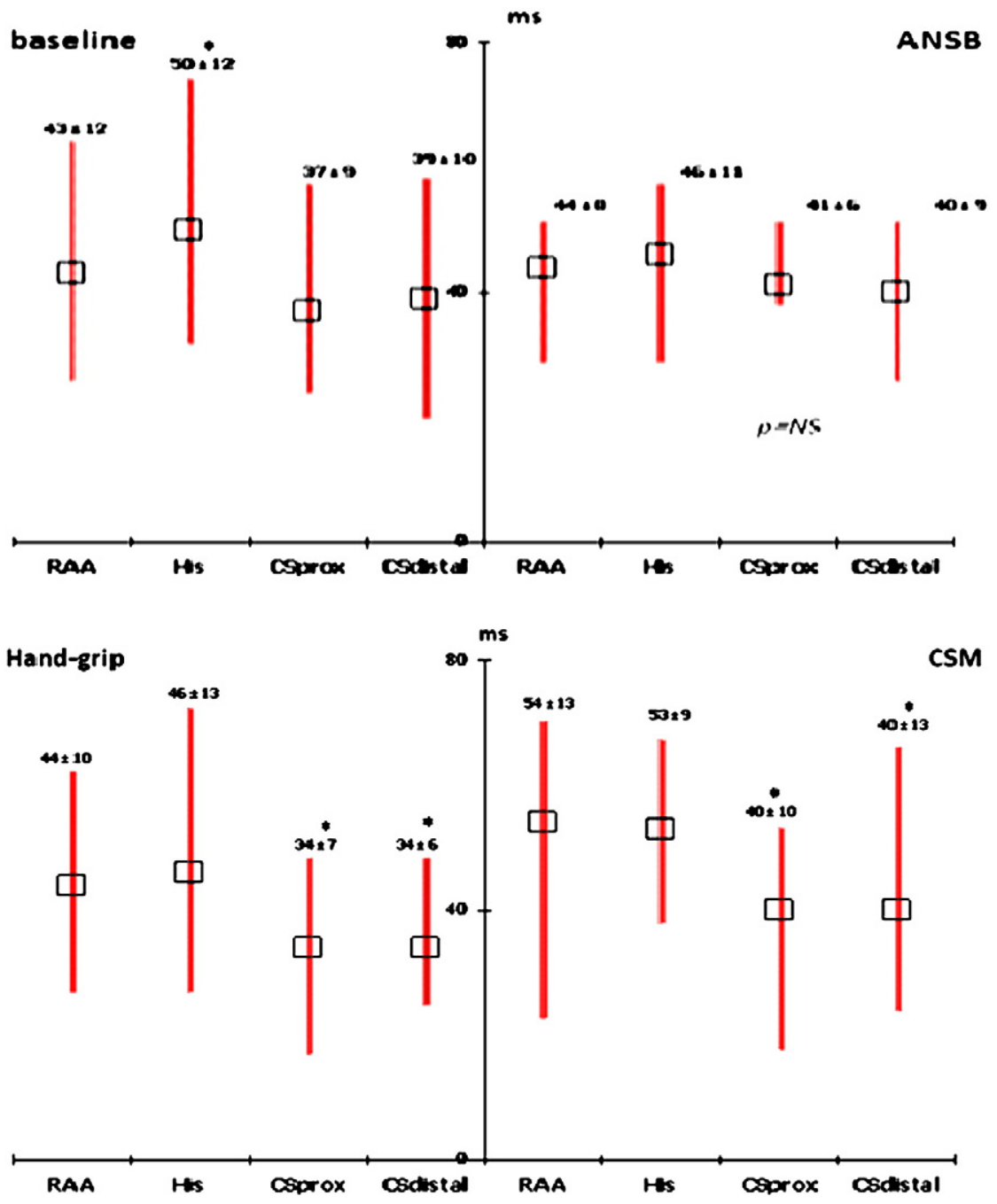

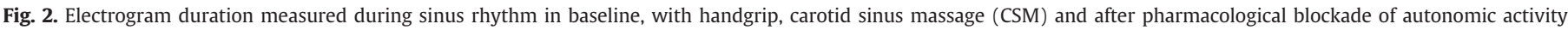

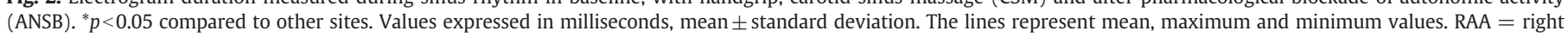
atrial appendage; His $=$ His position; CSprox $=$ proximal coronary sinus; CSdistal $=$ distal coronary sinus. 


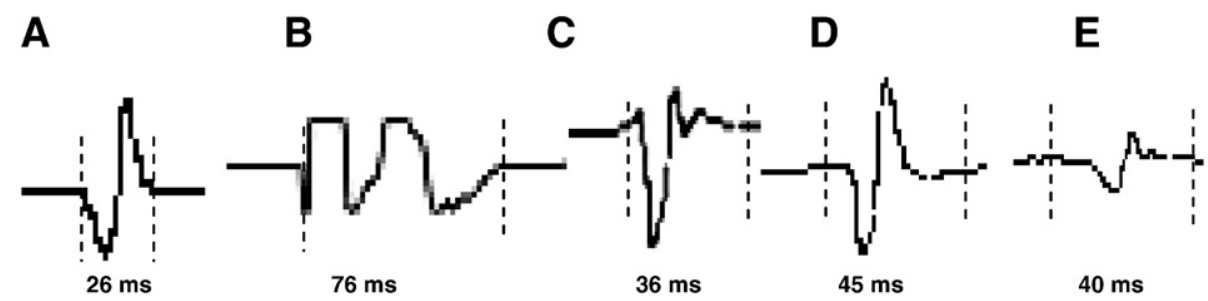

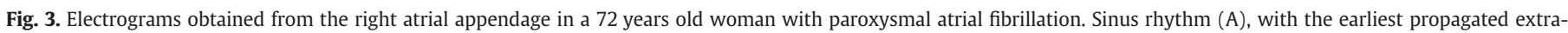
stimulus (B), during handgrip (C) and carotid sinus massage (D), and after pharmacological autonomic blockade (E).

occurrence of multiple reentry circuits within the atria, probably contributing to the electrophysiological substrate required for the presence of $\mathrm{AF}[3,6,18]$.

While prolongation of atrial conduction is a frequent finding in patients with AF $[19,20]$, the influence of autonomic activity in the atrial conduction intervals and local wave duration is incompletely explored. The present study characterized the IACT, the interACT and electrogram duration measured in different atrial recording sites in response to an extra-stimulus with a short coupling interval and during acute modulation of the ANS. There were no differences between the baseline characteristics of PAF patients and controls. However, the group with history of PAF showed longer IACT and RAA electrograms in baseline, compared with control patients. Furthermore, they showed significant atrial conduction delays and greater prolongation of atrial wave duration during early premature impulses delivered at the HRA. Also, fragmented atrial activity was identified in more patients with PAF than in control patients. These findings are consistent with previous studies who demonstrated greater delays in intra-atrial or inter-atrial conduction, and a higher incidence of atrial fragmentation in patients with PAF [6,20-22]. In fact, the presence of marked conduction delay during an atrial premature beat with a short coupling interval, combined with longer and fractionated electrograms is an important electrophysiological finding, compatible with the necessary conditions for the occurrence and maintenance of local reentry circuits. Focal repetitive activity, most frequently originated from pulmonary veins, plays an important role in the initiation of AF, particularly when combined with abnormal atrial impulse conduction, which appears to be pre-requisite for the maintenance of AF $[23,24]$. Heterogeneity of atrial conduction delay and the presence of local fragmented potentials have long been associated with the substrate for AF [25-27]. The greater prolongation of atrial activation times and of local wave duration with the earliest propagated extrastimulus, showed in our results, might contribute to explain why the mean coupling interval was significantly shorter for pulmonary veins discharges initiating AF than for discharges that did not in a recent study by Arentz et al. [28]. Therefore, a combination of atrial premature complexes with short coupling intervals and delayed activation of the atria may act as one component of the arrhythmogenic substrate for the vulnerability to PAF.

\subsection{Autonomic modulation of conduction parameters in PAF patients}

The supporting evidence of the impact of ANS activity in the electrophysiological properties of the atria and its role in the initiation and maintenance of AF has been mostly studied in experimental preparations. Little is known about the effects of acute stimulation or blockade of the ANS in atrial conduction and electrogram duration during electrophysiological evaluation of patients with PAF. In our data, obtained from patients with clinical history of PAF, the interACT and LAAT were significantly prolonged during CSM in the group with inducible sustained AF, supporting the notion that the substrate of AF is associated with conduction abnormalities of the atria, which can be more pronounced during vagal stimulation and contribute to the maintenance of AF. Although conduction abnormalities in PAF have been associated with increased age, atrial dilation and stretch, fibrosis, changes in the expression levels of connexins and electrophysiological remodeling [2,3,5-7,24], acute autonomic modulation seems to influence atrial conduction properties in patients with PAF.

It has been known that vagal stimulation shortens the atrial ERP and increases dispersion of atrial refractoriness [8,9,12,13]. Although both vagal and sympathetic stimulations could produce significant reductions on ERP, vagal stimulation appears more arrhythmogenic in promoting AF [29]. One reason could be related with the lengthening of atrial conduction time during vagal activity, that results in a pronounced wavelength shortening (ERP $\mathrm{x}$ conduction velocity), which would promote AF maintenance.

In the present study, we evaluated atrial electrograms duration during autonomic stimulation and after pharmacological autonomic blockade. Electrograms duration increased slightly in RAA and His during CSM, but no significant differences were obtained during acute autonomic modulation. However, when compared to baseline recordings, differences in atrial wave duration between the recording sites became more pronounced during ANS maneuvers and were abolished after ANS blockade. Although the impact of autonomic stimulation appears to be modest in the induction of $\mathrm{AF}$ (68.8\% during ANS maneuvers vs. 50\% after ANS blockade), it is possible that the electrogram duration heterogeneity and local conduction delay produced by autonomic modulation contribute to the initiation and maintenance of AF. Vagal stimulation has been found to result in a large regional heterogeneity of atrial electrograms, and there is evidence that the appearance of complex fractionated atrial electrograms during activation of the intrinsic cardiac autonomic neural elements reflects a change in the local electrophysiological properties [30,31]. In fact, differential areas of conduction velocity and dispersion of electrogram characteristics may provide a substrate for functional reentry, creating a suitable environment for $\mathrm{AF}[32,33]$.

There have been limited data on autonomic influences in the characteristics of electrograms during sinus rhythm. Guo et al., in a canine model, found that vagal stimulation shortened the electrogram duration in ischemic myocardium zone in the right atrium, whereas sympathetic stimulation did not alter electrogram duration [34]. In a previous analysis of atrial electrograms during sinus rhythm in patients with PAF, electrograms with $\geq 4$ deflections and duration $\geq 40$ ms were associated with a parasympathetic response during AF ablation [35]. The explanation for this finding was related with local effects of acetylcholine in atrial tissue, causing conduction block between adjacent fiber bundles. Recently, in a different study, complex fractionated atrial electrograms, representing slow conduction areas or pivoting points in reentry circuits, were induced by local application of varying concentrations of acetylcholine or by injecting acetylcholine into the anterior right ganglionated plexi, providing evidence that ANS activity may induce changes in local atrial conduction [36].

There is a great need for experimental and clinical studies to better understand the relationship between the dynamic changes in atrial electrogram morphology and autonomic innervation and its role in the maintenance of AF. 


\section{Study limitations}

Although it was possible to identify slight changes in atrial activation times and wave duration during acute autonomic modulation, the study included a small number of patients. However, all patients acted as their own controls to enable comparison of the parameters during stimulation and after blockade of the ANS. Although obtaining high density recordings by using multipolar catheters with better spatial resolution from several simultaneous right and left atrial sites could give more precise results, allowing a better comprehension of the problem, transseptal punctures for the use of left atrial catheters were not justifiable in a preliminary investigative study in humans. Another concern is that despite the confirmation of HG and CSM effects based on frequency domain spectral analysis, direct stimulation of sympathetic and parasympathetic nerves would have improved the results.

\section{Conclusions}

The presented study demonstrated that atrial conduction times, electrograms duration and fractionated activity are increased in patients with PAF when compared with control patients, suggesting that conduction abnormalities in the atria contribute to the arrhythmogenic substrate for AF. Also, acute vagal stimulation prolonged interACT and LAAT in patients with inducible sustained $\mathrm{AF}$ and ANS modulation influenced the heterogeneity of atrial electrograms duration in the recording sites. These should be taken into consideration in future studies in order to better understand the dynamic phenomena involved in the onset and perpetuation of AF episodes.

\section{Acknowledgement}

The authors of this manuscript have certified that they comply with the Principles of Ethical Publishing in the International Journal of Cardiology [37].

\section{References}

[1] Friberg J, Scharling H, Gadsbøll N, Jensen GB. Sex-specific increase in the prevalence of atrial fibrillation (The Copenhagen City Heart Study). Am J Cardiol Dec 15 2003;92(12):1419-23.

[2] Fuster V, Ryden LE, Canmon DS, et al. ACC/AHA/ESC 2006 guidelines for the management of patients with atrial fibrillation-executive summary. Eur Heart J 2006;27:1979-2030.

[3] Nattel S. Atrial electrophysiology and mechanisms of atrial fibrillation. J Cardiovas Pharmacol Ther 2003;8(1):S5-S11.

[4] Haissaguerre M, Jais P, Shah DC. Spontaneous initiation of atrial fibrillation by ectopic beats originating in the pulmonary veins. N Engl J Med 1998:339:659-66.

[5] Nattel S, Burstein B, Dobrev D. Atrial remodeling and atrial fibrillation. Mechanisms and implications. Circ Arrhythm Electrophysiol 2008;1:62-73.

[6] Pytkowski M, Jankowska A, Maciag A, et al. Paroxysmal atrial fibrillation is associated with increased intra-atrial conduction delay. Europace 2008;10: 1415-20.

[7] Nattel S, Shiroshita-Takeshita A, Cardin S, Pelletier P. Mechanisms of atrial remodeling and clinical relevance. Curr Opin Cardiol 2005;20:21-5.

[8] Olshansky B. Interrelationships between the autonomic nervous system and atrial fibrillation. Prog Cardiovasc Dis Jul-Aug 2005;48(1):1409-17.

[9] van den Berg MP, Hassink RJ, Baljé-Volkers C, Crijns HJGM. Role of the autonomic nervous system in vagal atrial fibrillation. Heart March 2003;89(3):333-5.

[10] Bettoni M, Zimmermann M. Autonomic tone variations before the onset of paroxysmal atrial fibrillation. Circulation June 11, 2002;105(23):2753-9.
[11] Amar D, Zhang H, Miodownik S, Kadish AH. Competing autonomic mechanisms precede the onset of postoperative atrial fibrillation. J Am Coll Cardiol October 2003;42(7):1262-8

[12] Chen PS, Tan AY. Autonomic nerve activity and atrial fibrillation. Heart Rhythm March 2007;4(3 Suppl):S61-4.

[13] Chen J, Wasmund SL, Hamdan MH. Back to the future: the role of the autonomic nervous system in atrial fibrillation. Pacing Clin Electrophysiol 2006;29:413-21.

[14] Miyamoto K, Nakao K, Seto S, et al. Abnormal right atrial electrograms predict the transition to chronic atrial fibrillation in paced patients with sick sinus syndrome. PACE 2004;27:644-50

[15] Cozma D, Mornos C, Pescariu S, Petrescu L, Lighezan D, Dragulescu S. Electrophysiological and echocardiographic parameters predisposing to atrial fibrillation in patients with a structurally normal heart. Kardiol Pol 2006;64: 143-50.

[16] Oliveira M, Silva N, Feliciano J, et al. Effects of stimulation and blockade of the autonomic nervous system on atrial refractoriness in patients with lone paroxysmal atrial fibrillation. Rev Port Cardiol 2009;28(6):1-16.

[17] Friedman HS, Sinha B, Tun A, et al. Zones of atrial vulnerability. Relationships to basic cycle length. Circulation 1996;94:1456-64.

[18] Oliveira M, da Silva MN, Timoteo AT, et al. Inducibility of atrial fibrillation during electrophysiologic evaluation is associated with increased dispersion of atrial refractoriness. Int J Cardiol August 2009;136(2):130-5.

[19] Platonov PG, Mitrofanova LB, Chireikin LV, Olsson SB. Morphology of inter-atrial conduction routes in patients with atrial fibrillation. Europace 2002;4:183-92.

[20] O'Donnell D, Bourke JP, Furniss SS. Interatrial transseptal electrical conduction: comparison of patients with atrial fibrillation and normal controls. J Cardiovasc Electrophysiol November 2002;13:1111-7.

[21] Cozma D, Kalifa J, Lighezan D, et al. Mechanism of atrial fibrillation: decremental conduction, fragmentation, and ectopic activity in patients with drug resistant paroxysmal atrial fibrillation and structurally normal heart. PACE 2005;28: S115-9.

[22] Tai CT, Chen SA, Tzeng JW, et al. Prolonged fractionation of paced right atrial electrograms in patients with atrial flutter and fibrillation. J Am Coll Cardiol 2001;37:1651-7.

[23] Rha S, Kim Y, Hong M, et al. Mechanisms responsible for the initiation and maintenance of atrial fibrillation assessed by non-contact mapping system. Int J Cardiol Feb 2008;124(2):218-26.

[24] Chaldoupi SM, Loh P, Hauer RNW, Bakker JMT, van Rijen HVM. The role of connexin40 in atrial fibrillation. Cardiovasc Res 2009, doi:10.1093/cvr/cvp203.

[25] Papageorgiou P, Monahan K, Boyle NG, et al. Site-dependent intraatrial conduction delay: relationship to initiation of atrial fibrillation. Circulation 1996;94:384-9.

[26] Saksena S, Giorgberidze I, Mehra R, et al. Electrophysiology and endocardial mapping of induced atrial fibrillation in patients with spontaneous atrial fibrillation. Am J Cardiol 1999;83:187-93.

[27] Ehrlich JR, Nattel S. Electrophysiological basis of atrial fibrillation. In: Schwartzman D, Zenati MA, editors. Innovative management of atrial fibrillation. 1st ed. Blackwell Publishing; 2005. p. 3-18.

[28] Arentz T, Haegeli L, Sanders P, et al. High-density mapping of spontaneous pulmonary vein activity initiating atrial fibrillation in humans. J Cardiovasc Electrophysiol January 2007;18:31-8.

[29] Liu L, Nattel S. Differing sympathetic and vagal effects on atrial fibrillation in dogs: role of refractoriness heterogeneity. Am J Physiol 1997;42(2):805-16.

[30] Vigmond E, Tsoi V, Kuo S, Yin Y, Trayanova N, Pagé P. Using atrial electrograms to estimate vagal influence. Heart Rhythm May 2005;2(5):S179 Supplement.

[31] Lin J, Scherlag B, Zhou J, et al. Autonomic mechanism to explain complex fractionated atrial electrograms (CFAE). J Cardiovasc Electrophysiol Nov 2007;18: 1197-205.

[32] Liuba I, Walfridsson H. Focal atrial tachycardia: increased electrogram fractionation in the vicinity of the earliest activation site. Europace July 2008, doi:10.1093/ europace/eun192.

[33] Makati K, Alsheikh-Ali A, Garlitski A et al. Advances in mechanisms of atrial fibrillation: structural remodeling, high-frequency fractionated electrograms, and reentrant AF drivers. J Interv Card Electrophysiol 2008;23:45-9.

[34] Guo H, Euler D, Wang Z, Olshansky B. Autonomic influences in atrial ischemia: vagally mediated atrial conduction improvement. Int J Cardiol September 1997;61 (2):157-63.

[35] Lellouche N, Buch E, Celigoj A, et al. Functional characterization of atrial electrograms in sinus rhythm delineates sites of parasympathetic innervation in patients with paroxysmal atrial fibrillation. J Am Coll Cardiol 2007;50:1324-31.

[36] Lin J, Scherlag BJ, Zhou J, et al. Autonomic mechanism to explain Complex Fractionated Atrial Electrograms (CFAE). J Cardiovasc Electrophysiol Nov 2007;18: 1197-205.

[37] Coats AJ. Ethical authorship and publishing. Int J Cardiol 2009;131:149-50. 